\title{
Structural Synthesis of 2R1T Type Mechanisms for Minimally Invasive Surgery Applications
}

\author{
A.Yaşır ${ }^{1}$ and G. Kiper $^{2}$ \\ ${ }^{1}$ Izmir Institute of Technology,Izmir,Turkey, e-mail: abdullahyasir@iyte.edu.tr \\ ${ }^{2}$ Izmir Institute of Technology, Izmir, Turkey, e-mail: gokhankiper@iyte.edu.tr
}

\begin{abstract}
Assistive and operative manipulators allow easier and more precise operations for minimally invasive surgery. Such manipulators often have a pivot point at the incision port on the patient's body, so the manipulator should have a remote center of motion. This study presents the structural synthesis of a non-parasitic 3-dof manipulator with 2R1T motion pattern to be used as a remote center of motion mechanism for minimally invasive surgery applications. The manipulators of various kinematic structure are evaluated considering criteria such as possibility of construction of the mechanism for remote center of motion, ease of dynamic balancing, number of links, structural symmetry, the number of actuators connected to the base and decoupling of the joint inputs and the output motion of the platform.
\end{abstract}

Keywords: Minimally invasive surgical manipulator, structural synthesis, non-parasitic motion, remote center of motion

\section{Introduction}

The use of robotic devices in surgical applications is becoming widespread day by day [2]. Some of these devices are used directly in operations while others are being developed as assistive devices. Compared with manual surgical operations, robot operated surgeries appear to be more precise [4]. Surgical robots are usually used in minimally invasive surgery (MIS) applications. MIS is performed with a surgical tool inserted through a small hole (incision port) into the patient's body. Such operations can be completed in less time, with less pain and less blood loss compared to conventional surgery. The post-operative process also results in faster recovery and smaller surgical scars [6].

The necessity of moving through an incision port in MIS requires that the robot should have a pivot point at the port. Remote-center-of-motion (RCM) is a point which remains stationary with respect to the base of the manipulator without necessitating any joints at that point such that the end-effector of the manipulator can rotate about and slide through this point. The RCM of a manipulator can be ob- 
tained by properly designing the mechanical structure, or by using a kinematically redundant manipulator and ensuring RCM by control. Mechanical RCMs are more reliable and considered more suitable for clinical practice compared to nonmechanical ones [7].

In MIS, a surgical tool can have at most four degrees-of-freedom (dof) through the incision port: yaw, pitch, roll and heave [7]. However, yaw, pitch and heave movements are sufficient for endoscope movements [8]. This study follows Kong and Gosselin's [3] structural synthesis methods in order to classify manipulators, end-effectors of which are capable of non-parasitic yaw, pitch and heave motions, i.e. 2R1T (two rotational, one translational dof) motion. The results are comparable with Li and Hervé's [5] results for classifying non-parasitic 1T2R manipulators, which are the kinematic inversion of the 2R1T case. Recently Huang et al. [1] also worked on RCM mechanisms with 2R1T motion, where they have similar results for synthesizing the legs, but the manipulators they obtained are few in number. After the classification, proper mechanical structures are compared according to design criteria for MIS applications with RCM.

\section{Structural Synthesis}

A manipulator with $2 \mathrm{R} 1 \mathrm{~T}$ motion can have various kinematic structures. The simplest kinematic structure would be an RRP (R: revolute, P: prismatic) serial manipulator where the $\mathrm{R}$ axes intersect each other and the $\mathrm{P}$ direction is perpendicular to the plane defined by the $\mathrm{R}$ axes. Also several types of hybrid kinematic structures can be used such as: type 1) the first R of the 2R1T motion is serially connected to a 2-dof parallel kinematic chain (PKC) for the RT motion; type 2) $2 \mathrm{R}$ motion is obtained with a PKC while the $\mathrm{T}$ motion is connected serially; type 3) second R motion is obtained with a PKC while the first $\mathrm{R}$ and the $\mathrm{T}$ motion are connected serially. Examples for serial and hybrid kinematic structures for orienting (2R) manipulators are presented in [9].

In this section, the structural synthesis for 3-dof parallel manipulators (PM) for $2 \mathrm{R} 1 \mathrm{~T}$ motion pattern is performed. The virtual chain (VC) corresponding to the 2R1T motion pattern is RRP. The motion and constraints of a kinematic chain are respectively represented by twist systems and wrench systems in screw theory [3]. Then, the wrench system for an $\mathbf{R R P}=\mathrm{PKC}$ is a $2 \mathrm{~F}_{0}-1 \mathrm{~F}_{\infty}$-system (Fig. 1a), where $\mathrm{F}_{0}$ and $\mathrm{F}_{\infty}$ represent 0 -pitch and $\infty$-pitch wrenches, respectively.

An RRP VC is illustrated in Fig. 1b. $\mathrm{u}$ - and v-axes represent the R joint axes of the $\mathrm{VC}$ and intersect at the pivot point $\mathrm{D}$. W-axis is along the direction of the $\mathrm{P}$ joint and passes through the pivot point. The u-axis lies on the XY-plane. u-axes attached to different legs may have different directions. v-axis is perpendicular to the $\mathrm{u}$-axis, while $\mathrm{w}$-axis is perpendicular to the v-axis. In Fig. 1b, when both yaw and pitch angles are equal to zero, $\mathrm{u}$ - and v-axes lie on the XY-plane and w-axis coincides with the Z-axis of the XYZ frame attached to the base. 
a.

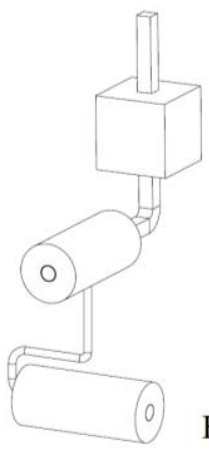

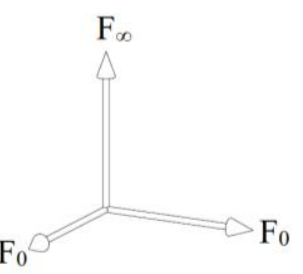

b.

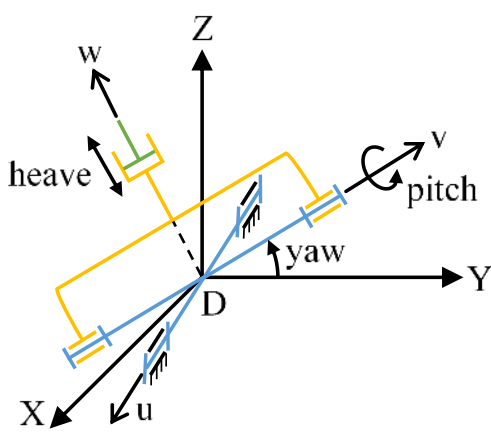

Fig. 1 a. $2 \mathrm{~F}_{0}-1 \mathrm{~F}_{\infty}$-system. b. Description of the RRP VC

\subsection{Decomposition of the wrench system of the PKC}

Combinations of leg constraint degrees $\left(\mathrm{c}^{1}, \mathrm{c}^{2}, \mathrm{c}^{3}\right.$ for the three legs) for 3-legged 3dof $(\mathrm{M}=3)$ PKCs are given in Table $1 . \Delta$ is the total degree of overconstraint of the PKC such that $\mathrm{c}^{1}+\mathrm{c}^{2}+\mathrm{c}^{3}=\Delta+\mathrm{M}$. The number of single dof joints, $\mathrm{f}$, in a leg is calculated with the formula $\mathrm{f}=(6-\mathrm{c})+\mathrm{M}$, where $\mathrm{c}$ is either of $\mathrm{c}^{1}, \mathrm{c}^{2}, \mathrm{c}^{3}$.

Table 1. Combinations of $\mathrm{c}^{\mathrm{i}}$ for 3-legged 3-dof PKCs

\begin{tabular}{|c||c|c|c|c|c|c|c|c|c|c|c|c|c|c|c|c|}
\hline$\Delta$ & $\mathbf{6}$ & $\mathbf{5}$ & \multicolumn{2}{|c|}{$\mathbf{4}$} & \multicolumn{3}{|c|}{$\mathbf{3}$} & \multicolumn{3}{|c|}{$\mathbf{2}$} & \multicolumn{3}{|c|}{$\mathbf{1}$} & \multicolumn{3}{|c|}{$\mathbf{0}$} \\
\hline \hline $\mathbf{c}^{\mathbf{1}}$ & 3 & 3 & 3 & 3 & 3 & 3 & 2 & 3 & 3 & 2 & 3 & 2 & 2 & 3 & 2 & 1 \\
\hline $\mathbf{c}^{\mathbf{2}}$ & 3 & 3 & 3 & 2 & 3 & 2 & 2 & 2 & 1 & 2 & 1 & 2 & 1 & 0 & 1 & 1 \\
\hline $\mathbf{c}^{3}$ & 3 & 2 & 1 & 2 & 0 & 1 & 2 & 0 & 1 & 1 & 0 & 0 & 1 & 0 & 0 & 1 \\
\hline
\end{tabular}

Table 2. Combinations of leg-wrench systems for 3-legged $\mathbf{R R P}=$ PKCs

\begin{tabular}{|c|c|c|c|c|c|c|c|c|c|c|c|c|c|c|c|c|}
\hline$\Delta$ & 6 & 5 & & \multicolumn{4}{|c|}{4} & \multicolumn{9}{|c|}{3} \\
\hline $\mathbf{c}^{1}, \mathbf{c}^{2}, \mathbf{c}^{3}$ & $3,3,3$ & 3,3, & & 3,3 , & & & & & $3,3,0$ & & 3,2 & & & & $, 2,2$ & \\
\hline $2 F_{0}-1 F_{\infty}$ & 3 & 2 & 2 & 2 & 2 & 1 & & & 2 & 1 & 1 & 1 & 1 & 0 & & 0 \\
\hline $1 F_{0}-1 F_{\infty}$ & 0 & 1 & 0 & 0 & 0 & 2 & & 1 & 0 & 1 & 1 & 0 & 0 & 3 & 2 & 1 \\
\hline $2 F_{0}$ & 0 & 0 & 1 & 0 & 0 & 0 & & 1 & 0 & 0 & 0 & 1 & 1 & 0 & 1 & 2 \\
\hline $1 F_{0}$ & 0 & 0 & 0 & 1 & 0 & 0 & & 0 & 0 & 1 & 0 & 0 & 1 & 0 & 0 & 0 \\
\hline $1 F_{\infty}$ & 0 & 0 & 0 & 0 & 1 & 0 & & $\underline{0}$ & 0 & 0 & 1 & 1 & 0 & 0 & 0 & 0 \\
\hline 0 & 0 & 0 & 0 & 0 & 0 & 0 & & 0 & 1 & 0 & 0 & 0 & 0 & & 0 & 0 \\
\hline
\end{tabular}

\begin{tabular}{|c|c|c|c|c|c|c|c|c|c|c|c|c|c|c|c|c|c|c|c|c|c|c|}
\hline$\Delta$ & \multicolumn{10}{|c|}{2} & \multicolumn{8}{|c|}{1} & \multicolumn{4}{|c|}{0} \\
\hline $\mathbf{c}^{1}, \mathbf{c}^{2}, \mathbf{c}^{3}$ & 3,2 & & & 1,1 & & & & 2, & & & & 1, & 2,2 & & & 2, & & & $3,0,0$ & & & $1,1,1$ \\
\hline $2 F_{0}-1 F_{\infty}$ & 1 & 1 & 1 & 1 & 1 & 0 & 0 & 0 & 0 & 0 & 1 & 1 & 0 & 0 & 0 & 0 & 0 & 0 & 1 & 0 & 0 & 0 \\
\hline $1 F_{0}-1 F_{\infty}$ & 1 & 0 & 0 & 0 & 0 & 2 & 0 & 2 & 1 & 1 & 0 & 0 & 2 & 1 & 1 & 0 & 1 & 0 & 0 & 1 & 0 & 0 \\
\hline $2 \mathbf{F}_{\mathbf{0}}$ & 0 & 1 & 0 & 0 & 0 & 0 & 2 & 0 & 1 & 1 & 0 & 0 & 0 & 1 & 0 & 1 & 0 & 1 & 0 & 0 & 1 & 0 \\
\hline $1 F_{0}$ & 0 & 0 & 2 & 0 & 1 & 1 & 0 & 0 & 1 & 0 & 1 & 0 & 0 & 0 & 2 & 0 & 1 & 1 & 0 & 1 & 0 & 2 \\
\hline $1 F_{\infty}$ & 0 & 0 & 0 & 2 & 1 & 0 & 1 & 1 & 0 & 1 & 0 & 1 & 0 & 0 & 0 & 2 & 1 & 1 & 0 & 0 & 1 & 1 \\
\hline 0 & 1 & 1 & 0 & 0 & 0 & 0 & 0 & 0 & 0 & 0 & 1 & 1 & 1 & 1 & 0 & 0 & 0 & 0 & 2 & 1 & 1 & 0 \\
\hline
\end{tabular}


The platform wrench system is a $2 \mathrm{~F}_{0}-1 \mathrm{~F}_{\infty}$-system, so the legs may have one of the following systems: $2 \mathrm{~F}_{0^{-}}-1 \mathrm{~F}_{\infty^{-}}, 1 \mathrm{~F}_{0^{-}}-1 \mathrm{~F}_{\infty^{-}}, 2 \mathrm{~F}_{0^{-}}, 1 \mathrm{~F}_{0^{-}}, 1 \mathrm{~F}_{\infty^{-}}$or 0 -system. The wrench system of the moving platform is simply the linear combination of those of all the legs [3]. So, combinations of leg-wrench system which do not have two $\mathrm{F}_{0}$ - and one $\mathrm{F}_{\infty}$-system in total fail to produce the platform wrench system and are not feasible. Feasible combinations are listed in Table 2.

\subsection{Type synthesis and assembly of the legs}

2F-1F $\mathbf{F}_{\infty}$-system: There should be $\mathrm{f}=(6-\mathrm{c})+\mathrm{M}=(6-3)+3=6$ joints in a virtual loop (VL). Since there are already 3 joints in the RRP VC, there should be 3 joints on the leg. In order to obtain a $2 \mathrm{~F}_{0}-1 \mathrm{~F}_{\infty}$-system, two coaxial $\left(3 \mathrm{~F}_{0}-2 \mathrm{~F}_{\infty}\right.$ system) and one codirectional $\left(2 \mathrm{~F}_{0}-3 \mathrm{~F}_{\infty}\right.$-system) compositional units (CUs) are combined (Table 3.3 in [3]). Coaxial or codirectional CUs are denoted by ()L, where the $\mathrm{R}$ or $\mathrm{P}$ joints are written inside the parenthesis. A coaxial CU can include one or more $\mathrm{R}$ joints with coincident axes. A codirectional $\mathrm{CU}$ can include one or more $\mathrm{P}$ joints with the same direction. Together with the RRP VC (or more specifically $\left.(\mathbf{R})_{\mathrm{L}}(\mathbf{R})_{\mathrm{L}}(\mathbf{P})_{\mathrm{L}} \mathrm{VC}\right)$, there are no feasible solutions for a VL, because the leg would have multiple coaxial $\mathrm{R}$ joints or multiple codirectional $\mathrm{P}$ joints. However, the VC itself can be used as a leg. Leg structure alternatives are $(R)_{L}(R)_{L}(P)_{L}$ and $(R)_{L}(R R)_{A}$, where ()$_{A}$ represents a parallel axis CU. Two or three legs of this type cannot be used in the PKC, because the centers of the universal (U) joints of multiple legs would necessarily be at the pivot point, hence not resulting in a RCM. So the columns in Table 2 with more than one $2 \mathrm{~F}_{0}-1 \mathrm{~F}_{\infty}$-system can be disregarded.

1F-1F $\infty$-system: A loop is composed of a coaxial CU and a planar CU (denoted by ()$\left._{E}\right)$. There are $f=(6-c)+M=(6-2)+3=7$ joints in the loop, 4 of which are on the leg. A planar CU has at least two joints, at least one of which is an R joint and all links move along parallel planes. There can be at most two $\mathrm{P}$ joints and more than one coaxial $\mathrm{R}$ joints is not allowed in the leg, because otherwise the leg has internal mobility. Since the R axes in the VC are orthogonal, the coaxial unit should definitely be a part of the VC. Also a part of the coaxial chain needs to be in the leg as well, because otherwise we have a coplanar 4-joint leg, which has internal mobility. So, the only possible structure for the VL is $\left((\mathrm{R} R)_{\mathrm{L}} \mathbf{R P E}\right)_{\mathrm{E}}$, where E stands for a planar joint. The VC joints are represented with bold letters in the VL in order to distinguish them from the joints of the leg. In practice, the E joint may be constructed as a planar RRR, PRR, RPR, RRP, PPR, PRP or RPP chain. $(R)_{L}$ part being inside the ()E parenthesis means that the coaxial $R$ joint can be positioned arbitrarily in between any two joints of the planar chain. Practically we do not prefer an unactuated $\mathrm{P}$ joint, so we disregard the solutions with multiple $P$ joints in a leg. The feasible legs are $\left((R)_{L} R R R\right)_{E},\left((R)_{L} R R P\right)_{E},\left((R)_{L} R P R\right)_{E}$ and $\left((R)_{L} P R R\right)_{E}$. The first three are equivalent to URR, URP and UPR legs, respectively. For $\left((R)_{L} P R R\right)_{E}$, if the $(R)_{L}$ axis is along the $\mathrm{P}$ direction, this specific case corresponds to a CRR chain. 


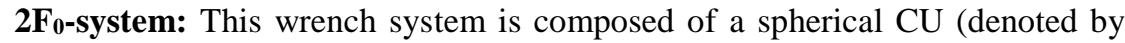
()$\left._{S}\right)$ and a codirectional CU. There are $\mathrm{f}=(6-\mathrm{c})+\mathrm{M}=(6-2)+3=7$ joints in the VL. A spherical CU includes at least two R joints and a codirectional CU includes at least one $\mathrm{P}$ joint. Two or more codirectional $\mathrm{P}$ joints in the leg are not feasible in practice. With these conditions, the only possible structure for the VL is $(\mathrm{SRR})_{\mathrm{S}}(\mathbf{P P})_{\mathrm{L}}$, where $\mathrm{S}$ stands for spherical joint. So the leg has a SP structure, which can have $(\mathrm{RRR})_{\mathrm{S}}(\mathrm{P})_{\mathrm{L}}$ form in practice.

1F 0 -system: There are $\mathrm{f}=(6-\mathrm{c})+\mathrm{M}=(6-1)+3=8$ joints in the VL of this wrench system. In Table 3.4 in [3], six different ways are given to obtain a $1 \mathrm{~F}_{0}$ system: (a) a planar CU + a spherical CU, (b) two spherical CUs with distinct centers, (c) inserting two coaxial CUs into a single-loop kinematic chain (SLKC) composed of a planar CU, (d) inserting two coaxial CUs into a SLKC composed of a spherical CU, (e) inserting a coaxial CU and a codirectional CU into a SLKC composed of a spherical CU and (f) inserting two codirectional CUs into a SLKC composed of a spherical CU. In case (a), the only possible SLKCs are in the form of $(R R R)_{S}(R P E)_{E}$. So, leg structures can be in the form of UE. The center of the U joint should be on the u-axis and the plane of the $U$ joint should not be parallel to the $\mathrm{u}$-axis. Plane of the E joint should be perpendicular to the v-axis. (b) and (d) cases are not feasible because of the $\mathbf{P}$ joint in the $\mathrm{VC}$. The only possible structure of VL for case $(c)$ is $\left((R)_{L}(R R)_{L} R P E\right)_{E}$. So, leg structure is $\left((R)_{L}(R)_{L} E\right)_{E}$. The axis of the $1^{\text {st }}(R)_{L}$ is along the $u$-axis; the axis of the $2^{\text {nd }}(R)_{L}$ is along the virtual $\mathbf{P}$ direction; plane of the E joint should be perpendicular to the V-axis; $(\mathrm{R})_{\mathrm{L}}$ 's can be distributed in the $\mathrm{E}$ joint. For case (e), the possible structures for VL are $(\mathrm{RRRRR})_{\mathrm{S}}(\mathbf{P P})_{\mathrm{L}}(\mathrm{R})_{\mathrm{L}}$ and $\left((\mathrm{RR})_{\mathrm{L}} \mathbf{R}(\mathrm{PP})_{\mathrm{L}} \mathrm{RRR}\right)_{\mathrm{S}}$. Thus, the leg structure can be $S(R)_{L}(P)_{L}$, where the center of the $S$ joint is on the pivot point, the axis of the $(R)_{L}$ does not pass through the pivot point and direction of $(\mathrm{P})_{\mathrm{L}}$ is along the $\mathrm{W}$-axis. Or alternatively the leg structure can be $\left((\mathrm{R})_{\mathrm{L}} \mathrm{S}(\mathrm{P})_{\mathrm{L}}\right)_{\mathrm{S}}$, where the axis of $(\mathrm{R})_{\mathrm{L}}$ is along the $\mathrm{u}$-axis, the center of the $\mathrm{S}$ joint is on the $\mathrm{v}$-axis and direction of $(\mathrm{P})_{\mathrm{L}}$ is along the w-axis. There are two possibilities for case $(f)$ : $\left(\operatorname{SRR}(\mathbf{P P})_{\mathrm{L}}(\mathrm{P})_{\mathrm{L}}\right)_{\mathrm{S}}$ (corresponds to leg structure $\left(\operatorname{RRR}(\mathrm{P})_{\mathrm{L}}(\mathrm{P})_{\mathrm{L}}\right)_{\mathrm{S}}$, which is not desirable due to two $\mathrm{P}$ joints in the leg) and $\left(\operatorname{SRRR}(\mathbf{P})_{\mathrm{L}}(\mathrm{P})_{\mathrm{L}}\right)_{\mathrm{S}}$ (corresponds to leg structure $\left(\mathrm{SR}(\mathrm{P})_{\mathrm{L}}\right)_{\mathrm{S}}$, which is not feasible due to internal mobility of the spherical $4 \mathrm{R}$ in the leg).

1F $\infty$-system: In this system, there are $\mathrm{f}=(6-\mathrm{c})+\mathrm{M}=(6-1)+3=8$ joints in the VL. For obtaining such a wrench system, we have two cases: (a) combining two parallel axis CUs or planar CUs or (b) inserting a coaxial CU into the SLKC composed of a parallel axis $\mathrm{CU}$ or planar CU. For case (a), using planar CUs is favorable over using parallel axis CUs due to constructional ease, so only planar CUs will be considered. Possible structures for the VL with two planar CUs are $(. . \mathbf{R})_{\mathrm{E}}(\mathbf{R P} . .)_{\mathrm{E}}$ and $(. . \mathbf{R})_{\mathrm{E}}(\mathbf{R P} . .)_{\mathrm{E}}$, where each dot represents an $\mathrm{R}$ or $\mathrm{P}$ joint. Plane of the first ()$_{\mathrm{E}}$ is perpendicular to the $\mathrm{u}$-axis and plane of the second ()$_{\mathrm{E}}$ is perpendicular to the v-axis. So the leg structure is $(. .)_{\mathrm{E}} \mathrm{E}$ or $\mathrm{E}(. .)_{\mathrm{E}}$ where .. may be RR, RP or PR. For case (b), possible structures for the VL have the form of $\left((R \mathbf{R})_{\mathrm{L}} \mathbf{R P} \ldots\right)_{\mathrm{A}}$. So the leg can be $\left((\mathrm{R})_{\mathrm{L}} \mathrm{RRRP}\right)_{\mathrm{A}},\left((\mathrm{R})_{\mathrm{L}} \mathrm{RRPR}\right)_{\mathrm{A}},\left((\mathrm{R})_{\mathrm{L}} \mathrm{RPRR}\right)_{\mathrm{A}}$ or $\left((R)_{L} P R R R\right)_{A}$. $(R)_{L}$ is coaxial with the $u$-axis and the remaining $R$ joint axes are all parallel to the $\mathrm{V}$-axis. 
0-system: In this system, there are no constraints for the dof of the leg and any 6-dof chain is suitable. SPU can be an example.

Possible leg configurations are summarized in Table 3.

Table 3. Leg alternatives for each system

\begin{tabular}{|c|c|}
\hline System & Leg Structure Alternatives \\
\hline $2 \mathrm{~F}_{0}-1 \mathrm{~F}_{\infty}$ & $(\mathrm{R})_{\mathrm{L}}(\mathrm{R})_{\mathrm{L}}(\mathrm{P})_{\mathrm{L}} ;(\mathrm{R})_{\mathrm{L}}(\mathrm{RR})_{\mathrm{A}}$ \\
\hline $1 \mathrm{~F}_{0}-1 \mathrm{~F}_{\infty}$ & $\begin{array}{l}(\mathrm{R})_{\mathrm{L}} \mathrm{E}\left((\mathrm{R})_{\mathrm{L}} \text { is co-axial with } 1^{\text {st }} \mathrm{R} \text { joint axis of the } \mathrm{VC}\right):\left((\mathrm{R})_{\mathrm{L}} \mathrm{RRR}\right)_{\mathrm{E}} \text { or URR; } \\
\left((\mathrm{R})_{\mathrm{L}} \mathrm{PRR}\right)_{\mathrm{E}}(\text { may be } \mathrm{CRR}) ;\left((\mathrm{R})_{\mathrm{L}} \mathrm{RPR}\right)_{\mathrm{E}} \text { or UPR; }\left((\mathrm{R})_{\mathrm{L}} \mathrm{RRP}\right)_{\mathrm{E}} \text { or URP }\end{array}$ \\
\hline $2 \mathrm{~F}_{0}$ & SP (The center of S joint should be coincident with the pivot point D) \\
\hline $1 \mathrm{~F}_{0}$ & $\begin{array}{l}\text { a) UE (center of } U \text { joint is on u-axis; plane of } U \text { joint is not parallel to u-axis; plane of } \\
\text { E joint is perpendicular to v-axis): } S(R R)_{E}, S(R P)_{E}, S(P R)_{E}, U(P R R)_{E} \\
\text { c) }\left((R)_{L}(R)_{L} E\right)_{E}\left(\text { axis of } 1^{\text {st }}(R)_{L} \text { is along u-axis; axis of } 2^{\text {nd }}(R)_{L} \text { is parallel to w-axis; }\right. \\
\left.\text { plane of joint } E \text { is perpendicular to v-axis; }(R)_{L} \text { 's can be distributed in } E \text { joint }\right) \\
\text { e) } S(R)_{L}(P)_{L}\left(\text { center of } S \text { joint is on pivot point; axis of }(R)_{L} \text { does not pass through }\right. \\
\text { pivot point; direction of }(P)_{L} \text { is along w-axis) or }\left((R)_{L} S(P)_{L}\right)_{S} \text { (axis of }(R)_{L} \text { is along u- } \\
\text { axis; center of } S \text { joint is on v-axis; direction of }(P)_{L} \text { is along w-axis) }\end{array}$ \\
\hline $1 \mathrm{~F}_{\infty}$ & $\begin{array}{l}\text { 1) }(. .)_{\mathrm{E}} \mathrm{E}\left(\text { plane of }()_{\mathrm{E}} \text { is perpendicular to u-axis; plane of } \mathrm{E} \text { joint is perpendicular to v- }\right. \\
\text { axis): }(\mathrm{RR})_{\mathrm{E}}(\mathrm{RRR})_{\mathrm{E}},(\mathrm{RR})_{\mathrm{E}}(\mathrm{RRP})_{\mathrm{E}},(\mathrm{RR})_{\mathrm{E}}(\mathrm{RPR})_{\mathrm{E}},(\mathrm{RR})_{\mathrm{E}}(\mathrm{PRR})_{\mathrm{E}} ;(\mathrm{RP})_{\mathrm{E}}(\mathrm{RRR})_{\mathrm{E}} \text {; } \\
(\mathrm{PR})_{\mathrm{E}}(\mathrm{RRR})_{\mathrm{E}} ; \mathrm{E}(\mathrm{RR})_{\mathrm{E}}:(\mathrm{RRR})_{\mathrm{E}}(\mathrm{RR})_{\mathrm{E}},(\mathrm{RRP})_{\mathrm{E}}(\mathrm{RR})_{\mathrm{E}},(\mathrm{RPR})_{\mathrm{E}}(\mathrm{RR})_{\mathrm{E}},(\mathrm{PRR})_{\mathrm{E}}(\mathrm{RR})_{\mathrm{E}} \text {; } \\
(\mathrm{RRR})_{\mathrm{E}}(\mathrm{RP})_{\mathrm{E}} ;(\mathrm{RRR})_{\mathrm{E}}(\mathrm{PR})_{\mathrm{E}} \\
\text { 2) }\left((\mathrm{R})_{\mathrm{L} R R} \mathrm{RR}\right)_{\mathrm{A}},\left((\mathrm{R})_{\mathrm{L}} \mathrm{RRPR}\right)_{\mathrm{A}},\left((\mathrm{R})_{\mathrm{L}} \mathrm{RPRR}\right)_{\mathrm{A}},\left((\mathrm{R})_{\mathrm{L}} \mathrm{PRRR}\right)_{\mathrm{A}}\end{array}$ \\
\hline 0 & Example: SPU \\
\hline
\end{tabular}

$\mathbf{R R P}=$ PKCs can be generated by assembling three of the alternative leg structures given in Table 3. For each six alternative leg-wrench systems in Table 3, a representative leg is selected and possible assemblies according to Table 2 are constructed in a CAD program. Some of the assemblies result in 4-dof platform motion due to dependency of the leg wrench systems, so they are disregarded. For the assemblies that satisfy the 2R1T motion which can be used as an RCM mechanism, the options for the three joints to be actuated are evaluated. Preferably, the actuated joints should be connected to the base.

\section{Design Evaluation}

For some of the assemblies obtained in Section 2 the legs share the first $\mathrm{R}$ axes on the base, hence they actually have a hybrid type 1 kinematic structure. All the manipulators obtained in Section 2 are evaluated along with hybrid types 2 and 3 and serial assemblies. All alternatives are compared with each other considering the following evaluation criteria with their weights, w: ease of dynamic balancing $(\mathrm{w}=3)$; number of links $(\mathrm{w}=1)$; structural symmetry $(\mathrm{w}=1)$; decoupling of the inputs and the output $2 \mathrm{R} 1 \mathrm{~T}$ motion $(\mathrm{w}=2)$; and the number of actuators connected to the base $(\mathrm{w}=2)$. For grading, the following considerations were taken into account: For ease of dynamic balancing, grade of legs with prismatic joints (if unavoidable) and/or spherical sub-chains was kept low. Less number of links was 
considered as an advantage. Structural symmetry grade is determined based on whether there are same types of legs and the legs can be positioned in opposite sides. Decoupling of the inputs and the output 2R1T motion was graded based on how many inputs directly correspond to an $\mathrm{R}$ or $\mathrm{T}$ motion of the end-effector. Finally, more number of actuators that can be connected to the base was considered to be in favor.

The evaluation chart is too big to be presented here. The kinematic structure with the highest grade in the evaluation chart has a $2\left(1 \mathrm{~F}_{0}\right.$-system $)$ and $1\left(1 \mathrm{~F}_{0}-1 \mathrm{~F}_{\infty^{-}}\right.$ system) leg structure. A CAD model of such a system designed as an RCM manipulator for transsphenoidal surgery is depicted in Fig. 2. The PM in Fig. 2 has 2URRR-URR kinematic structure. For this PM, the R joint axes on the base should be intersecting at the pivot point, but the angles between the axes are arbitrary. The $\mathrm{R}$ axes of the URRR legs connected to the endoscope should be concurrent along the w-axis. Although all legs in Fig. 2 seem to have identical structure, one of the legs has one less $\mathrm{R}$ joint (about the w-axis), hence the part of the leg after the second $\mathrm{R}$ joint is rigidly connected to the endoscope.

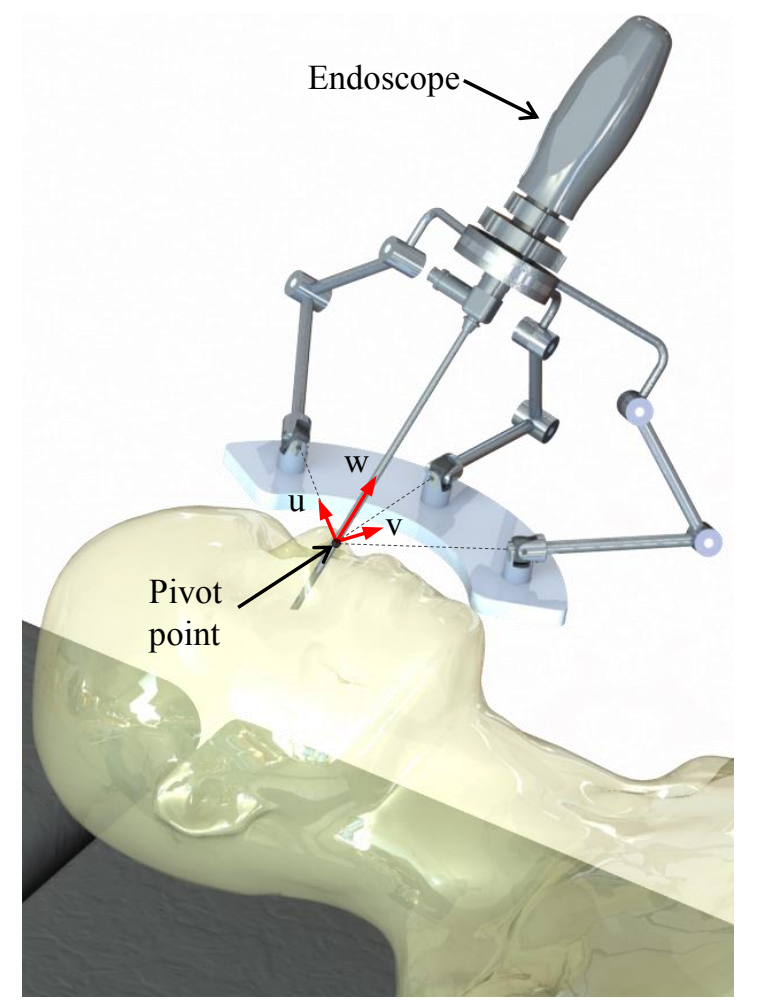

Fig. 2 Conceptual CAD model of an RCM manipulator for transsphenoidal surgery 


\section{Conclusions}

This study deals with the structural synthesis of a non-parasitic 3-dof manipulator with 2R1T motion pattern to be used as an RCM mechanism for MIS applications. For the synthesis of the PMs, the constraint based type synthesis method of Kong and Gosselin [3] is used. The resulting PMs together with manipulators with hybrid and serial kinematic structure are evaluated. The key evaluation criteria are possibility of construction of the mechanism for RCM and ease of dynamic balancing. The best option is evaluated to be a PM with $2\left(1 \mathrm{~F}_{0}\right.$-system $)$ and $1\left(1 \mathrm{~F}_{0^{-}}\right.$ $1 \mathrm{~F}_{\infty}$-system) leg structure which has a total degree of overconstraint of $\Delta=1$. The future studies include optimization of the dimensions of the PM for a desired dexterous workspace, dynamic balancing and constructional design.

Acknowledgments This study is funded by The Scientific and Technological Research Council of Turkey (grant number 115E726).

\section{References}

1. Huang, L., Guang, C., Yang, Y. and Su, P.: Type Synthesis of Parallel 2R1T Remote Center of Motion Mechanisms Based on Screw Theory. MATEC Web of Conf., 95, (2017) doi: $10.1051 /$ matecconf $/ 20179508009$

2. Kim, K. C. (Ed.): Robotics in General Surgery. Springer (2014)

3. Kong, X. and Gosselin, C.: Type Synthesis of Parallel Mechanisms. Springer (2007)

4. Kuo, C. H. and Dai, J. S.: Robotics for minimally invasive surgery: a historical review from the perspective of kinematics. In: International Symposium on History of Machines and Mechanisms, pp. 337-354 (2009) doi: 10.1007/978-1-4020-9485-9

5. Li, Q. and Hervé, J. M.: 1T2R parallel mechanisms without parasitic motion. IEEE Trans. Robot., 26(3), 401-410 (2010) doi: 10.1109/TRO.2010.2047528

6. Li, J., Xing, Y., Liang, K. and Wang, S.: Kinematic design of a novel spatial remote center-ofmotion mechanism for minimally invasive surgical robot. J. Med. Devices, 9(1), 011003, (2015) doi: 10.1115/1.4028651

7. Liu, S., Chen, B., Caro, S., Briot, S., Harewood, L. and Chen, C.: A cable linkage with remote centre of motion. Mech. Mach. Theory, 105, 583-605 (2016) doi: 10.1016/j.mechmachtheory.2016.07.023

8. Taniguchi, K., Nishikawa, A., Sekimoto, M., Kobayashi, T., Kazuhara, K., Ichihara, T., Kurashita, N., Takiguchi, S., Doki, Y., Mori, M. and Miyazaki, F.: Classification, design and evaluation of endoscope robots. In: Baik, S. H. (Ed.), Robot Surgery. InTech (2010) doi: $10.5772 / 6893$

9. Teichgräber, C., Müglitz, J. and Berger, M.: Guiding linkages with remote centre of rotation for thermal cutting processes. In: Dede, M. İ. C., İtik, M., Lovasz, E-C. and Kiper, G., Mechanisms, Transmissions and Applications: Proceedings of the Fourth MeTrApp Conference 2007. Springer (2017) 